\title{
Estudio comparativo randomizado de la eficacia clínico-bacteriológica de mecillinam versus Cotrimexazole en Shigellosis
}

Dres. Valeria Prado J. ${ }^{1,2}$ Jacob Cohen V. ${ }^{1,2}$ Abdala Harun M. ${ }^{2}$ TM. Sra. Ximena Aguire C. ${ }^{1}$ TM: Srta. Cristina Diaz J. ${ }^{1}$

\begin{abstract}
Forty intants, with Shigellosis were studied. Twenty of them, selected at random were treated with Mexill1$\mathrm{nam}$ in dusis of $45 \mathrm{mg}$ ( $\mathrm{Kg}$ day dusing 48 hours via $\mathrm{IM}$, and then, with $60 \mathrm{mg}$ (Kg/day per os by three days.

The remained group of twenty infants were treated with Cotrimexazole (Bactrim ${ }^{\mathrm{H}}$ ) in dosis of timethoprin 6 $\mathrm{mg}$ ( $\mathrm{Kg} / \mathrm{day}$ vía IM the first $48 \mathrm{~h}$ hrs and then by oral via until complete five days.

Clinical (characteristics and frecuency of stools, weight gain and temperature) Bactenological tdaily stools cultures) and Biochemical pareneters (CPT, AF pre and post-trealment) were evaluated while antimicrobial therapy was adininistered.

The analisis of results demostrated, that both antimicrobial agents had similar cinical-bacteriological eticacy, being satisfactory in $80 \%$ of cases. Nevertheless it was observed a Cotrimexazole superionity but without statistic significancy.
\end{abstract}

Como resultado de la constante investigación en busca de nuevas drogas antimicrobianas, recientemente se ha sintetizado un nuevo derivado B lactámico: la Mecillinam, que posee una cadena lateral totalmente diferente a otros derivados del ácido 6 amino penicilánico, es un ácido 6-B amidino penicilínico, lo que le confiere ventajas desde el punto de vista microbiológico, ya que actía interfiriendo en la síntesis de la pared celular bacteriana de un modo diferente a Penicilinas y Cefalosporinas. ${ }^{1,2}$

Es así como numerosos trabajos han demostrado que la actividad in vitro de este antibiótico es excelente frente a bacterias Gram negativas, principalmente de la Familia Enterobacteriaceae, resultando superior a la actividad de Ampicilina y Amoxicilina. 3, 4, 5,6,7

Existen ademăs evidencias de los buenos resultados clínicos obtenidos en el tratamiento de infecciones del aparato urinario ${ }^{89}$ y en fiebre tifoidea. ${ }^{\text {IU, } 11}$

La presente investigación tiene como objetivo evaluar desde el punto de vista clínico y bacteriológico la eficacia de Mecillinam en el tratamiento de la Shigellosis en niños, estableciendo una comparación con Cotrimexazol (Bactrim, ${ }^{\mathrm{R}}$ ) droga que en nuestro medio ha demostrado buena efectividad tanto in vivo como in vitro trente a Shigella. ${ }^{12}$

\footnotetext{
${ }^{1}$ Universidad de Chile-Facultad de Medicina - Santiago Oriente - Unidad de Microbiologia.

2Servicio de Pediatuía - Hospital Luis Calvo Mackenna.
}

Estudios in vitro han demostrado que específicamente frente a Shigella la Mecillinam es más activa que Ampicilina. ${ }^{6.7}$

La Shigellosis en lactantes no es una enfermedad autolimitada a diferencia de lo observado en edades posteriores como to hemos podido corroborar en nuestro medio ${ }^{13} y$ en ocasiones conduce a diarrea prolongada, por lo cual es importante el uso de un antimicrobiano eficaz que asegure niveles plasmáticos adecuados.

Un objetivo adicional fue el de ensayar un esquema terapéutico que incluye administración parenteral del antimicrobiano durante las primeras $\mathbf{4 8}$ horas y continuar por vía oral hasta completar $\overline{5}$ días de tratamiento.

\section{MATERLAL Y METODO}

Se estudiaron 40 nin̄os que ingresaron al Hospital Calvo Mackenna por cuadros de diarrea aguda con o sin síndrome disentérico, entre los meses de febrero de 1979 y febrern de 1980 , a los cuales se les aisló Shigella de las deposiciones. Los coprocultivos se realizaron tomando una muestra de deposición recién emitida con tórula de madera y transportada en medio de Cary Blair.

Las muestras fueron sembradas en medios selectivos: Agar SS y Mac Conkey.

Las colonias lactosa negativas se estudiaron en la siguiente serie de medios diferenciales: TSI, LIA, MIO, citrato de Simons y caldo peptonado más urea; aquellas colonias con las características bioquímicas de Shigella se aglutinaron con sueros 
grupales polivalentes y posterionmente estas cepas fueron referidas al Centro Nacional de Enterobacterias del Instituto Bacteriológico para su confirmación, siendo todas ratificadas.

Un grupo de 20 pacientes, siguiendo una tabla de randomización, recibió Mecillinam inicialmente en dosis de $15 \mathrm{mg} / \mathrm{kg}$-p/ dosis $(45 \mathrm{mg} / \mathrm{kg}$ p/día) c/8 hrs. por vía intramuscular durante $48 \mathrm{hrs}$ y posteriormente se continuó con la forma oral esterificada Pivmecillinan $20 \mathrm{mg} / \mathrm{kg}$-p/dosis $(60$ $\mathrm{mg} / \mathrm{kg}$-p/dia) cada $8 \mathrm{hrs}$ por otras 72 horas.

Un segundo grupo de 20 pacientes se trató con Cotrimexazole (Bactrim ${ }^{\mathrm{R}}$ Roche) en dosis de $3 \mathrm{mg}$ de Trimetoprim por $\mathrm{Kg} / \mathrm{dosis}$ ( $6 \mathrm{mg} / \mathrm{kg}$-p día) cada 12 lirs por vía intramuscular, seguido de igual dosis y frecuencia por vía oral hasta completar 5 dias.

Durante el curso del tratamiento se efectuaron Coprocultivos diarios (total 5) y además se tomó un Coprocultivo 72 hrs después de finalizado el tratamiento para evaluar el tiempo de erradicación de Shigella del intestino con los dos antimicrobianos en estudio.

En forma paralela al estudio de enterobacterias patógenas, se sembraron las deposiciones en Agar Saboureaud para observar multiplicación de levaduras.

Se estudió la sensibilidad in vitro de las cepas de Shigella aisladas por las técnicas de difusión en disco: Kirby-Bauer en medio Welcotest y por la técnica de dilución en placa, determinándose la CIM en mcg/ml de los siguientes antimicrobianos: Mecillinam - Cotrimexazol - Ampicilina Amoxicilina-Gentamicina-Cloramfenicol.

En cada paciente en estudio se evaluaron diariamente los siguientes parámetros clínicos: fiebre, deshidratación, característica y frecuencia de las deposiciones, peso, pulso, presión arterial.

Al inicio y término del tratamiento se efectuaron los siguientes análisis bioquímicos: Hemograma, velocidad de eritrosedimentación, electrólitos plasmáticos, transaminasas glutámica pirúvjca, fosfatasas alcalinas, orina: examen quimico y microscópico.

No se utilizaron agentes antiespasmódicos ni absorbentes.

Para la evaluación se estableció una escala con cuatro categorías de respuesta clínico-bacteriológica definida como:

- Muy buena: deposiciones normales, coprocultivo negativo dentro de los 3 primeros días.

- Buena: normalización de las deposiciones con coprocultivo negativo entre el 4." o 5. día de tratamiento.

- Regular: al 5." día deposiciones normales con coprocultivo positivo para Shigella, o coprocultivo negativo con persistencia de la diarrea.

- Mala: al $5 .^{\circ}$ día de tratamiento, persistencia de la diarrea y coprocultivo positivo.

\section{RESULTADOS}

La Tabla N. I muestra las características clínicas del grupo estudiado. La distribución de las edades, sexo y la sintomatología clínica acompanante que era similar en ambos grupos de tratamiento.

La Tabla N. ${ }^{\circ} 2$ muestra los serotipos de ShigeIla aislados en los niños estudiados, todos pertenecientes al subgrupo flexnerii.

En la Tabla N. ${ }^{\circ} 3$ se detallan los resultados de los controles bacteriológicos de deposiciones. En el grupo Mecillinam persistía la Shigella al $50^{\circ}$ día de tratamiento en 1 caso y en un paciente se observó aparición de otra bacteria enteropatógena. En el grupo Cotrimexazole se erradicó Shigella en todos los pacientes y en 2 de ellos aparecieron otros enteropatógenos.

E] desarrollo de levaduras en ambos grupos fue similar.

La Taba N. 4 resume la respuesta clínicobacteriológica en ambos grupos de tratamiento. Se observó cierta superionidad del Cotrimexazole sobre Mecillinam aunque sin significancia estadística.

En la Tabla N. 5 se analizan las concentraciones inhibitorias mínimas de 6 antimicrobianos frente a las cepas de Shigella aisladas. El $92.5 \%$ de las cepas era sensible a Mecillinam y el $75 \%$ a Cotrimexazole, ambas drogas en estudio.

La Tabla N ${ }^{\circ} 6$ muestra las variaciones observadas en la CIM de Mecillinam frente a Shigella en medios con diferente concentración de electrólitos.

La Tabla N. 7 muestra las alteraciones de VHS y de la cifra de leucocitos en los niños estudiados.

En el cuadro N. 8 vemos las variaciones de algunos parámetros de funcionamiento hepático durante el tratamiento. En el grupo Mecillinam se observó un aumento significativo de transaminasas GP, pero dentro de los rangos normales.

En la Tabla N." 9 se detallan algunas alteraciones clinicas $y$ de laboratorio observadas que podrían atribuirse a la acción de las drogas en uso. 
Tabla N: I

Mecillinam versus Cotrimexazole en 40 casos de Shigellosis en nin̄os hospital Calvo Mackenna

Características Clínicas

\begin{tabular}{|c|c|c|c|}
\hline & $\begin{array}{c}\text { Mecillinam } \\
\text { N. }\end{array}$ & $\begin{array}{c}\text { Cotrimexazole } \\
\text { N. }{ }^{\circ}\end{array}$ & $\mathbf{P}$ \\
\hline $\begin{array}{l}1 \text { año } \\
\text { Edad } 1 \text { año } 1 \text { d. - } 2 \text { años } \\
2 \text { años } 1 \text { d. }-3 \text { años }\end{array}$ & $\begin{array}{r}15 \\
3 \\
2\end{array}$ & $\begin{array}{r}18 \\
2 \\
0\end{array}$ & $>0.20^{*}$ \\
\hline $\begin{array}{l}\text { Masculino } \\
\text { Sexo } \\
\text { Fernenino }\end{array}$ & $\begin{array}{l}8 \\
12\end{array}$ & $\begin{array}{l}11 \\
9\end{array}$ & $>0.50^{*}$ \\
\hline $\begin{array}{l}\text { Sindrome disentérico } \\
\text { Deshidratación } \\
\text { Fiebre }\left(37.5^{\circ} \mathrm{C} \text { ax. }\right) \\
\text { Alt. neurologgicas } \\
\text { Temesmo }\end{array}$ & $\begin{array}{r}16 \\
18 \\
12 \\
1 \\
2\end{array}$ & $\begin{array}{r}17 \\
14 \\
12 \\
1 \\
7\end{array}$ & $>0.20^{x}$ \\
\hline Infección enteral asociada & $2^{1}$ & $\theta^{2}$ & $>0.20^{*}$ \\
\hline
\end{tabular}

1. I ECEP; 1 ECEP + S. typhimurium

2. 4 ECEP: 1 S. typhim, 1 Ent. hystolitica

${ }^{*} \mathrm{~N} . \mathrm{S}$.

Tabla N. ${ }^{\circ} \mathbf{2}$

Mecillinam versus Cotrimexazole en 40 casos de Shigellosis en niños

Tipos de Shigellas

\begin{tabular}{|c|c|c|}
\hline Subgrupo & Serotipo & N.o Cepas \\
\hline & $1 . \mathrm{a}$ & 4 \\
\hline \multirow[t]{2}{*}{ Shigella } & 2.a & 14 \\
\hline & $2 b$ & 2 \\
\hline \multirow[t]{3}{*}{ flexnerii } & $3 . a$ & 10 \\
\hline & 6 Boyd 88 & 3 \\
\hline & sin tipificar & 7 \\
\hline Total & & 40 \\
\hline
\end{tabular}

Tabla N.03

Mecillinam versus Cotrirnexazole en 40 casos de Shigellosis en niños

Evaluación bacteriologica

\begin{tabular}{|c|c|c|c|c|c|}
\hline $\begin{array}{l}\text { Grupo } \\
\text { Tratamiento }\end{array}$ & $\begin{array}{c}\text { N." } \\
\text { total } \\
\text { casos }\end{array}$ & $\begin{array}{c}\text { Dias de } \\
\text { tratamiento }\end{array}$ & $\begin{array}{l}\text { Persiste } \\
\text { Shigella }\end{array}$ & $\begin{array}{c}\text { Otros } \\
\text { Enteropatógenos }\end{array}$ & $\begin{array}{l}\text { Levaduras } \\
-50 \mathrm{col} / \mathrm{plac} a\end{array}$ \\
\hline Mecillinam & 20 & $\begin{array}{l}\text { 3.er día } \\
5 .^{0} \text { día } \\
8 .^{0} \text { día }\end{array}$ & $\begin{array}{c}\text { N. casos } \\
0 \\
1 \\
0\end{array}$ & $\begin{array}{c}\text { N. casos } \\
1 \\
0 \\
0\end{array}$ & $\begin{array}{c}\text { N. casos } \\
8 \\
8 \\
9\end{array}$ \\
\hline Cotrimexazole & 20 & $\begin{array}{l}\text { 3er. día } \\
5 .^{\circ} \text { día } \\
8 .^{\circ} \text { día }\end{array}$ & $\begin{array}{l}0 \\
0 \\
0\end{array}$ & $\begin{array}{l}2 \\
2 \\
1\end{array}$ & $\begin{array}{l}12 \\
10 \\
10\end{array}$ \\
\hline
\end{tabular}

1 E. coli $086 \cdot 5$ typhimurium

2 E. coli $0127+$ E. coli $055+$ S. typhimurium 
Tabla N. 4

Evaluación Clinico - Bacteriológica

\begin{tabular}{|c|c|c|c|c|c|}
\hline \multirow[b]{2}{*}{ Respuesta } & \multicolumn{2}{|c|}{ Grupo Mecillinam } & \multicolumn{2}{|c|}{ Grupo Cotrimexazole } & \multirow[b]{2}{*}{$p$} \\
\hline & N. casos & $\%$ & N."casos & $\%$ & \\
\hline Muy Buena & 6 & 30 & 11 & 55 & $>0.10^{* *}$ \\
\hline Buena & 9 & 45 & 6 & 30 & \\
\hline Regular & 4 & 20) & 3 & 15 & \\
\hline Mala* & 1 & 5 & 0 & 0 & \\
\hline
\end{tabular}

*Shigella resistente + ECEP + S. typhimuriurı

** N.S.

Tabla N.05

Concentración inhibitoria mínima en $\mathrm{mcg} / \mathrm{ml}$ de 6 antimicrobianos frente a 40 cepas de Shigella Flexnerii

\begin{tabular}{|c|c|c|c|c|c|c|}
\hline \multirow[b]{2}{*}{ Antimicrobianos } & \multicolumn{3}{|c|}{ CIM mcg/ml } & \multirow[b]{2}{*}{$\geq 25$} & \multicolumn{2}{|c|}{ Cepas sensibles } \\
\hline & $\leq 0.78$ & $1.56-3.12$ & $6.25-12.5$ & & $\mathbf{N} .^{\sigma}$ & $\%$ \\
\hline Mecillinam & 36 & 1 & 2 & 1 & 37 & 92.5 \\
\hline Cotrimexazole & 17 & 13 & 9 & $\mathbf{I}$ & 30 & 75.0 \\
\hline Ampicilina & 23 & 13 & 2 & 2 & 38 & 95.0 \\
\hline Amoxicilina & 4 & 32 & 1 & 3 & 37 & 92.5 \\
\hline Cloramfenicol & 16 & 21 & 2 & $\mathbf{I}$ & 39 & 97.5 \\
\hline Gentamicina & 37 & 3 & 0 & 0 & 40 & 100.0 \\
\hline
\end{tabular}

Tabla N.6

Variaciones sensibilidad in vitro de Mecillinam en medios con diferente Osmolaridad

\begin{tabular}{|c|c|c|c|}
\hline Bacteria & $\begin{array}{c}\text { No } \\
\text { cepas }\end{array}$ & $\begin{array}{l}\text { Müller Hinton } \\
\left(\mathrm{H}_{2} \mathrm{O} \text { destilada }\right) \\
\text { CIM mcg/m] }\end{array}$ & $\begin{array}{c}\text { Welcotest } \\
\left(\mathrm{H}_{2} \mathrm{O} \text { bidestilada desmineralizada }\right) \\
\text { ClM mcg'ml }\end{array}$ \\
\hline Shigella & 13 & 0.19 & 0.09 \\
\hline \multirow{5}{*}{ flexnerii } & 3 & 0.79 & 0.09 \\
\hline & 1 & 0.19 & 0.045 \\
\hline & 1 & 6.25 & 1.56 \\
\hline & 1 & 6.25 & 3.12 \\
\hline & 1 & 50.0 & 6.25 \\
\hline
\end{tabular}

Total 20

Tabla N. ${ }^{\circ} \mathbf{7}$

Parámetros Bioquímicos

Grupo Cotrimexazole Grupo Mecillinan

\begin{tabular}{|c|c|c|c|c|c|c|c|c|}
\hline \multirow[b]{2}{*}{$V \mathrm{H} \mathrm{S} \mathrm{mm}$} & \multicolumn{2}{|c|}{ Pre - Trat } & \multicolumn{2}{|c|}{ Post-Trat } & \multicolumn{2}{|c|}{ Pre - Trat } & \multicolumn{2}{|c|}{ Post - Trat } \\
\hline & N. ${ }^{\circ}$ & $\%$ & No & $\%$ & N." & $\%$ & N: & $\%$ \\
\hline$\leq 10$ & 6 & 32 & 5 & 26 & 6 & 30 & 4 & 20 \\
\hline $11-30$ & 9 & 47 & 10 & 53 & 9 & 45 & 14 & 70 \\
\hline$* \geq 30$ & 4 & 21 & 4 & 21 & 5 & 25 & 2 & 10 \\
\hline
\end{tabular}

Leucocitos $x \mathrm{~mm}^{3}$

\begin{tabular}{lrrrrrrrr}
\hline $5.000 \cdot 10.000$ & 6 & 30 & 8 & 40 & 3 & 15 & 6 & 33 \\
$10.001-15.000$ & 10 & 50 & 9 & 45 & 9 & 45 & 10 & 55 \\
215.000 & 4 & 20 & 3 & 15 & 8 & 40 & 2 & 12 \\
\hline
\end{tabular}


Tabla N. ${ }^{\circ}$

Parámetros Hionuímicos

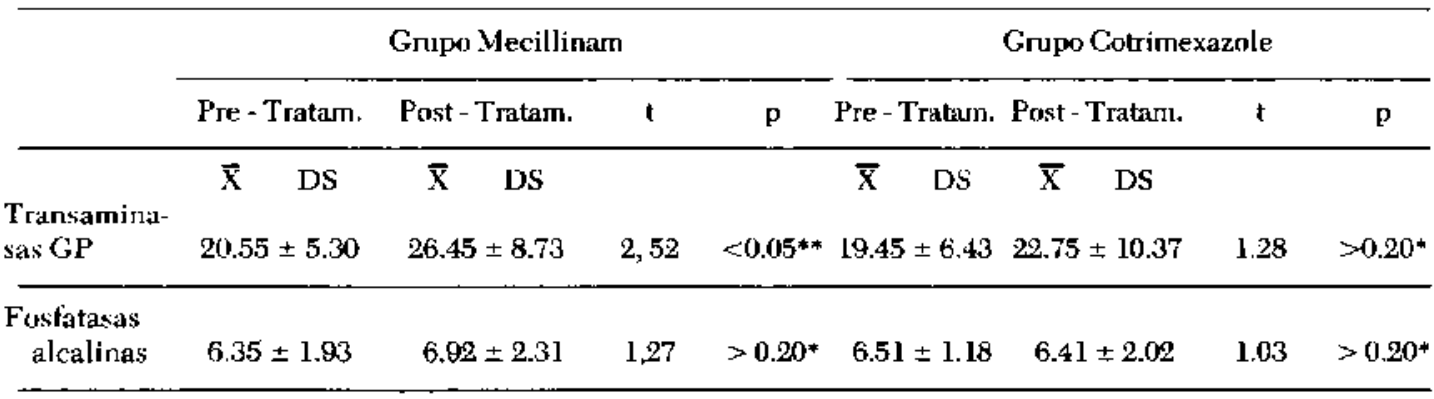

Tabla N.0 9

Mecillinam versus Cotrimexazole en 40 casos de Shigellosis en niños efectos adversos.

\begin{tabular}{|c|c|c|c|c|c|}
\hline \multirow[t]{2}{*}{$\begin{array}{c}\text { Manifestaciones clínicas } \\
\text { N. }{ }^{\circ} \text { casos }\end{array}$} & \multicolumn{5}{|c|}{$\begin{array}{l}\text { Alt. Laboratorio } \\
\text { N." casos }\end{array}$} \\
\hline & Vómitos & Reacc. cut. & $\mathrm{Hb}^{*}$ & $\mathrm{GPT}^{*}$ & Fosf. Alc.* \\
\hline Gnupo Cotrimexezole & 2 & 0 & 1 & l & 0 \\
\hline Grupo Mecillinam & 0 & 0 & 3 & 1 & 1 \\
\hline
\end{tabular}

\section{COMENTARIO}

Creemos de interés destacar algunas características'relevantes de la serie clínica en que se desarrolló el estudio.

Atrae la atención el alto porcentaje de pacientes con síndrome disentérico ( $80 \%$ ), lo cual no representa la incidencia habitual de aparición de disentería en las infecciones por Shigella en nuestro medio, que generalmente es de un $50 \%{ }^{1{ }^{13}}$ Por eso es necesario aclarar que se trata de una muestra diferente, pues para la pesquisa de Shigellosis se puso mayor énfasis en la toma de muestra en pacientes con deposiciones disentéricas.

Otras sintomatologías como fiebre y deshidratación se encontraron presentes en la frecuencia habitual. ${ }^{13}$

En este grupo de pacientes se puso interés especial en la constatación de tenesmo, signo que estaba presente en el $22,5 \%$ de los casos y que asociado a la existencia de síndrome disentérico es de gran ayuda clínica para sospechar unả Shigellosis. (Tabla N. ${ }^{\circ}$ ).
La existencia de infecciones enterales bacterianas mixtas se detectó en un $20 \%$ de los casos, fenómeno que hemos visto repetirse en estudios etiológicos de diarrea que hemos efectuado anteriormente ${ }^{14,}{ }^{15}$ y que es fácil de entender si se piensa que la fuente y mecanismos de infección son los mismos para los diferentes enteropatógenos. Es necesario considerar la posibilidad de infecciones mixtas para el manejo terapéutico adecuado de algunos cuadros diarreicos.

En cuanto a la edad de nuestros pacientes, como ya lo hemos comentado en trabajos anteriores, ${ }^{13}$ tradicionalmente vemos que la Shigellosis y en general las infecciones enterales bacterianas tienen un impacto mayor en nin̄os menores de 2 años, debido a factores de mayor susceptibilidad del huésped.

Respecto al objetivo central de nuestro trabajo, cual fue la evaluación de la eficacia clínico-bacteriológica de las dos drogas en estudio, podemos decir, de acuerdo a nuestros resultados, que el Cotrimexazol mostró cierta superioridad sobre la Mecillinam, con un $85 \%$ de respuesta Muy Buena 
o Buena frente a $\mathbf{7 5} \%$ observada con Mecillinam.

Sin embargo, esta diferencia no revistio signifjcación estadistica.

Observamos de esta manera una discrepancia entre la actividad in vivo e in vitro de la Mecillinam, la cual en el ląboratorio mostró mejor actividad que Cotrimexazole frente a las cepas de Shigella, retlejado en concentraciones inhibitorias minimas menores $y$ un porcentaje mayor de cepas sensibles, considerando que esta droga alcanza niveles plasmáticos que lluctúan entre 5-6 mcg/ml después de 1 hora de administrada. ${ }^{16}$

Esta discrepancia nos indica que la biodisponibilidad de la Mecillinam no es la óptima, quizás se podría explicar por la vida media de corta duración que tiene este antimicrobiano, aproximadamente 1 hora, ${ }^{16.13}$ lo cual con la frecuencia de administración c/8 hrs. utilizada en nuestro estudio no se logra una utílización efectiva de la droga en forma continua. Se podría dilucidar este punto efectuando curvas de inhibición sérica, en que se mide la capacidad bactericida del suero del paciente una hora antes y después de la administración del antibiótico, frente a la cepa del mismo paciente.

Erradicación de la Shigella de las deposiciones se logró en todos los casos menos en uno que recibió Mecillinam y la Shigella era resistente a dicha droga.

Proliferación de Levaduras (Candida) a nivel intestinal se observó aproximadamente en la mitad de los pacientes estudiados, sin diferencias en los dos grupos de tratamiento, que podríamos considerarlo como un parámetro que refleja la alteración de la flora intestinal comensal. Ningún paciente presentó en el curso del tratamiento evidencias clínicas de moniliasis.

Respecto a los estudios de sensibilidad efectuados con las cepas de Shigella aisladas, nos muestra que las Shigellas que se aislan en nuestro país siguen manteniendo una elevada sensibilidad a los distintos antimicrobianos. ${ }^{13}$

Al efectuar estos ensayos de sensibilidad in vitro es importante considerar la concentración de electrolitos del medio de cultivo, ya que un exceso de iones especialmente Caleio, disminuye la actividad in vitro de algunos antimicrobianos, especialmente importante en el caso de Mecillinam lo que queda evidenciado en la Tabla N. ${ }^{\circ} 6$.

Creemos de interés comentar los valores de la velocidad de eritrosedimentación observados en estos pacientes con Shigellosis. Como se puede apreciar (Tabla N. ${ }^{0} 7$ ), esta patologia no altera en forma importante este indice y en aquellos pacientes que presentaban VHS con cifras superiores a $30 \mathrm{~mm}$ en 1 hord, a la infección enteral se asociaban otros focos infecciosos especialmente a nivel del árbol urinario.

En cuanto a reacciones adversas, en el grupo tratado con Mecillinam se observó muy buena tolerancia de la droga, tanto de la forma parenteral como del derivado esterificado para uso oral: la Pivmecillinam. En este grupo observamos algunas alteraciones bioquimicas en cinco pacientes traducidas en disminución de la hemoglobina bajo cifras normales en 3 casos (aun cuando estos pacientes habian presentado síndrome disentérico), aumento discreto de las transaminasas en un paciente y de fosfatasas alcalinas en otro. La frecuencia con que se observaron alteraciones de estos índices coincide con lo comunicado por otros autores que han utilizado Mecillinam en pacientes adultos. ${ }^{17}$

En el grupo que recibió Cotrimexazole se observó intolerancia digestiva que se expreś́ por la aparición de vómitos en dos pacientes, aun cuando es dificil en una infección gastrointestinal atribuir con exactitud el síntoma vómito al medicamento administrado. Se detectó además disminución de la hemoglobina en un paciente y en otro un aumento de las transaminasas GP.

En ningún caso fue necesario suspender el tratamiento debido a estas alteraciones.

\section{CONCLUSION}

En nuestro medio al comparar la eficacia clínicobacteriológica de Mecillinam versus Cotrimexazole en niños con Shigellosis, se observaron resultados semejantes en ambos grupos, con respuestas satisfactorias en aproximadamente el $80 \%$ de los casos, con cierta superioridad del Cotrimexazole, aunque no significativo desde el punto de vista estadístico.

\section{RESUMEN}

Se estudiaron 40 nin̂os con Shigellosis, en su mayoría lactantes. En forma randomizada un grupo de 20 niños se trató con Mecillinam en dosis de 45 mgr Kg-p/día durante $48 \mathrm{hrs}$ por vía intramuscular y posteriormente $60 \mathrm{mgr} \mathrm{Kg-p/día} \mathrm{por} \mathrm{vía} \mathrm{oral}$ hasta completar 5 días. El segundo grupo de 20 niños recibió Cotrimexazole $\left(\right.$ Bactrim $^{\mathrm{R}}$ ) en dosis 
de 6 mgr de Trimetoprim ( $\mathrm{Kg}$-p/día) por vía intramuscular las primeras $48 \mathrm{hrs}$ y posteriormente se continuó por vía oral hasta totalizar 5 días.

En cada paciente se evaluaron diariamente, mientras duró la terapia antimicrobiana, parámetros clínicos como característica y frecuencia de las deposiciones, curva de peso, temperatura; Bacteriológicos, para lo cual se efectuaron coprocultivos diarios y Bioguimicos (transaminasas GP y fosfatasas alcalinas pre y post-tratamiento). El análisis de los resultados demostró que la eficacia clínica-bacteriológica de ambos antimicrobianos fue semejante, siendo satisfactoria en aproximadamente el $80 \%$ de los casos, observándose cierta superioridad del Cotrimexazole que no alcanzó a revestir significación estadística.

\section{REFERENCIAS}

${ }^{1}$ Matsuhaski, M. et al. Study of mechanism of action and development of resistance to a new amidino penicillin. J. Bacteriol. 117; 578-587, 1974.

${ }^{2}$ Sprett, B.G. The mechanism of action of mectlinam. J, Antmicrob. Chether 3 Suppl. B, 13-19, 1977.

3 Anderson, J.D. Mecillinam resistance in clinical practice, a review. J. Antrimicrobial. Chem. 3 Suppl. B, 89-96, 1977.

4 .Makao, M. al al. In vitro antibacterial activity of Mecillinam and Pivmecillinam. Chemotherapy 25, 1:61-86, 1977.

5 Nakazawa, 5. et al. Bactenological studies on pivmecillinam, new B-lactam antibiotics. Chemotherapy 25, 1: 28-45, 1977.

${ }^{6}$ Nets, H.C. Mecillinam, a novel penicillanic acid derivate with unsual activity aganist Gram-negative bacteria. Antímicrob. Agents. Chemother. 10, 793-799, 1976.

${ }^{7}$ Neu, H.C. Mecillinam - an amidino pirmicillin whill acts synerpisticelly with other B lactan compourd. J. Antimicrob. Chemother. 3 (Suppl. B), 43-52, 1977.

${ }^{B}$ Bresky, B. Randomized study comparing anoxizililli and pivmecithiman in adult aut patients presenting with syuptous of urinary infection. J. Antimicrob. Chemother. 3 Suppl. 8 , $121-127,1977$

${ }^{9}$ Clarke, P.D. \& Ceddes, A.M. Pivmecilinam in uriagry tract infections a correlation of urinary bactericidae with clinical efficacy. J. Antimicrob. Chemother. 3, 160-173, 1977.

${ }^{10}$ Clarke, P.D.; Geddes, A.M.; Mc Ghie, D. \& Walt, J.C. Mecillnanl: a new antibiotic for enteric fever. Brit. Med. J.: 14-15, 1976.

${ }^{11}$ Gedides, A.M. \& Clarke, P.D. The treatment of enteric fever with Mecillinam. J. Antimicrob. Chemother, 3 Supply, B, 101 102.

12 Prado, V.; Banfi, A. y Donoso, E. Evaluación clínico-bacteriological del uso de Cotrimexazole en el tratamiento de la Shigellosis en lactantes. Rev. Chilena Ped., 46; 25, 1975.

13 Prodo, V., Cohen, J y Barfi, A. Amoxicilina en el tratamiento de niños con Shigellosis, Rev. Chilena Ped., 50: 27, 1979.

14 Lynch, B.; Prado, V. y Reyes, H. Etiologia microbiana de la diarrea aguda con deshidratación en lactantes chilenos. 1 parte. VIl Congresa Latinoamericuno de Microbiologia. Buenos Aires, 1977.

${ }^{15}$ Prado, V,; Aeyes, H. y Lynch, B. Etiologia microbiana de la diarrea simple en lactantes chilenos. II parte. VII Congreso Latínoamericano de Microbiologia, Buenos Aires, 1977.

15 Mitchard, $M_{, ;}$Andrews, J; Kendal, MJ. \& Wise, R. Mecilli. nam serun levels following intravenous injection: a comparison with pivmecillinarn. J. Antimicr. Chemother. 3 (Suppl. B), 83-88, 1977 .

${ }^{17}$ Rohold, K.; Nielsen, B. and Kristensen, E. Pharmacokinetic studies with mecillinam and pivmecillinam. J. Antimicrob. Chemother. 3 Suppl, 13: $71-81,1977$. 